\title{
QUASISTATIC ISOTHERMAL EVOLUTION OF SHAPE MEMORY ALLOYS
}

\author{
SERGIO FRIGERI, PAVEL KREJČÍ, AND ULISSE STEFANELLI
}

\begin{abstract}
This note focuses on a three-dimensional phenomenological model for the isothermal evolution of a polycrystalline shape memory alloy. The model, originally proposed by Auricchio, Taylor, and Lubliner in 1997, is thermodynamically consistent and reproduces the crucial martensitic reorientation effect as well as the tension-compression asymmetric behavior of the material. We prove the existence of a weak solution of the corresponding quasistatic evolution problem by passing to the limit within a time-discretization procedure.
\end{abstract}

Key words: Shape Memory Alloys, Quasistatic evolution, Existence, Time-discretization.

AMS Subject Classification: 74C05, 35K55.

\section{INTRODUCTION}

Shape memory alloys (SMAs) are metallic alloys showing an amazing capability of recovering large deformations [26]. In the isothermal high-temperature regime SMAs exhibit the so-called superelastic behavior (also called pseudoelastic or even pseudoplastic): deformations up to $8 \%$ are fully recovered upon unloading (note that ordinary steels plasticize around 1\%). By allowing temperature changes, SMAs show also the celebrated shape memory effect: permanently deformed specimens can be forced back into the original undeformed configuration by a purely thermal treatment (heating).

At the microscopic level, SMAs experience abrupt and diffusionless stress- and temperaturedriven phase transitions between different configuration of the metallic lattices. In particular, a highly symmetric crystallographic phase called austenite (mostly cubic, predominant at higher temperatures) transforms into less symmetric phases called martensites (different variants due to symmetry breaking, energetically favorable at lower temperatures). By cooling down at zero stress a fully austenitic specimen below some critical temperature, the material develops a finely structured martensitic phase. Roughly speaking, martensitic variants combine in twins in order to minimize macroscopic motions. This structured phase is called multi-variant martensite (or twinned, non-oriented, self-accomodated). By keeping the temperature constant and applying an external stress, one specific martensitic variant turns out to be mechanically favorable and both the austenite and/or the multi-variant martensite progressively transform into a single-variant martensite (detwinned, oriented). This fact gives rise to a macroscopic deformation due to the specific asymmetry of the selected single martensitic variant. The latter phase transformation is of the first order (no latent heat) and the single-variant martensite (product phase) transforms back to austenite and/or multi-variant martensite (parent phase) upon unloading.

The special thermomechanical behavior of SMAs is at the basis of a variety of innovative applications from Aerospace, to Earthquake, to Biomedical Engineering and has been the subject of an intense research within the Materials Engineering community in recent years [19, 20]. Indeed, SMA behavior has been investigated at all scales (microscopic, mesoscopic with volume fractions, macroscopic) by means of a full menagerie of modeling perspectives [53] and with ambitions for different ranges of applicability (from single crystals to commercial devices). A collection of different macroscopic/phenomenological modeling options and some corresponding discussion is to be found in the papers [22, 23, 25, 27, 29, 34, 48, 35, 46, 49, 50, 54, 55], see also the survey [53]. 
We are here concerned with a 3D model of the isothermal, superelastic behavior of polycrystalline SMAs specimens originally advanced by AURICCHIO, TAYLOR, \& Lubliner [12] (ATL model in the following). The temperature of the body is assumed to be fixed (and suitably high) throughout and stress-induced reversible phase transformations between parent and product phases are considered. The main focus of the ATL model is toward a description of the martensitic reorientation phenomenon, that is the inelastic behavior determined from the progressive stress-driven reorientation of fully oriented, single-variant martensites. In particular, the inelastic deformation $\varepsilon^{\text {tr }}$ due to phase transformation is assumed in the form

$$
\varepsilon^{\operatorname{tr}}=\xi \frac{\partial F(\sigma)}{\partial \sigma}
$$

where $F$ is the so-called Drucker-Prager function of the stress $\sigma$. Note that the specific choice of $F$ will take into account the asymmetric response of the material in tension and compression. In the spirit of generalized plasticity models [36], the inelastic description of the material from (1.1) is complemented by directly prescribing a rate-independent evolution law for the single-variant martensite volume fraction $\xi$.

The ATL model is scalar: the actual phase structure of the material is completely encoded in the scalar internal variable $\xi$ and the martensitic reorientation phenomenon is described in (1.1) by assuming a priori that the relevant direction of $\varepsilon^{\text {tr }}$ is directly given by the stress $\sigma$ via $F$. This is of course a crude simplification with respect to the truly tensorial nature of superelasticity in polycrystals, some results in the direction of coping with a tensorial internal variable may be found in $[5,6,7,8,9,31,32]$. Still, the simplicity of the ATL model, its robustness with respect to discretizations [10], and its ability to capture experimental evidence are remarkably promising. The first mathematical treatment of the ATL model is given in [10] where the authors focus on the constitutive material equation and present an effective discretization algorithm. In particular, approximations are obtained by adapting to the current context the well-known return map algorithm in plasticity and sharp and explicit error bounds as well as numerical experiments are presented. The analysis of [10] has been extended and combined with a well-posedness theory for the related quasistatic one-dimensional equilibrium problem in [11]. In particular, time-discrete schemes with sharp error bounds have been obtained for both the stress and the displacement-driven case.

As the ATL model is restricted to the isothermal regime, the description of the shape memory effect is clearly out of reach. We shall however stress that the isothermal approximation shows often good accordance with experiments in real superelastic situations. This is particularly the case when the changes of the loading are comparably slow and the SMA body is thin at least one direction. In this case one can assume that the heat produced by the dissipative reorientation mechanism is (almost) immediately transported to the surrounding environment. Even in terms of energetics, experimental evidence shows that the influence of (irreversible) internal dissipation is very small compared with the (reversible) thermomechanical energy dynamics along loadingunloading cycles [47].

The main result of this paper is the proof of the existence of a weak solution of the quasistatic equilibrium problem in three space dimensions. The strategy of the proof is based on a timediscretization technique and the bottleneck of all the analysis is clearly the treatment of (1.1) for passage to limits in this product necessarily requires strong convergences. We achieve this by compactness and direct Cauchy arguments and, in particular, by including a deformationgradient term in the momentum balance equation. The key point of the convergence proof for the time-discretization is a dissipativity estimate. Despite the failure of monotonicity and the nonsmoothness in (1.1), the specific form of the evolution law for $\xi$ allows for a crucial bound on time derivatives (see (5.7) below).

We shall stress that, the ATL model is associative in the sense of classical plasticity theory [28]. In particular, the constitutive equation for the material can be expressed as a generalized 
balance between conservative and dissipative actions driven by potentials (see Subsection 2.6) and we are hence dealing with a so-called rate-independent system (see the survey [41] and the references therein). On the other hand, our existence result cannot be directly reduced to the by now classical theory of energetic solvability of such systems initiated by Mielke \& TheIL [40] and later developed by many Authors (see [18, 24, 33, 38, 39, 52] among others) for the involved potentials lack the required smoothness. This particularly motivates our ad hoc analysis.

\section{THE ATL MODEL}

We devote this section to introduce some notation and recall the basic features of the ATL model from [12]. Note that additional material on the ATL model can be found in $[3,4,36]$ where the interested reader is referred for remarks, outcome of numerical experiments, and comments on validation.

2.1. Tensors. Let $\mathbb{R}_{\mathrm{sym}}^{3 \times 3}$ denote the space of symmetric $3 \times 3$ tensors endowed with the natural scalar product $A: B \doteq \operatorname{tr}\left(A B^{\prime}\right)=A_{i j} B_{i j}$ (summation convention) and the norm $|A|^{2} \doteq A: A$. We decompose $\mathbb{R}_{\mathrm{sym}}^{3 \times 3}=\mathbb{R}_{\mathrm{dev}}^{3 \times 3} \oplus \mathbb{R} 1_{2}$ where $\mathbb{R}_{\mathrm{dev}}^{3 \times 3}$ is the deviatoric subspace of $\mathbb{R}_{\mathrm{sym}}^{3 \times 3}$ defined by $\operatorname{tr} A=0$ and $1_{2}$ is the identity 2-tensor. For all $u \in H_{\text {loc }}^{1}\left(\mathbb{R}^{3} ; \mathbb{R}^{3}\right)$ we let $\varepsilon(u) \doteq\left(\mathrm{D} u+\mathrm{D} u^{\prime}\right) / 2 \in$ $L_{\text {loc }}^{2}\left(\mathbb{R}^{3} ; \mathbb{R}_{\text {sym }}^{3 \times 3}\right)$ denote the standard symmetric gradient. Given any $A, B \in \mathbb{R}^{3 \times 3 \times 3}$ (3-tensors), we define the triple contraction product $A \because B$ as $A \because B \doteq A_{i j k} B_{i j k}$.

2.2. Reference configuration and boundary displacement. Let the reference configuration of the body be the non-empty, bounded, and connected open set $\Omega \in \mathbb{R}^{3}$ with Lipschitz continuous boundary $\Gamma$. Moreover, let $Q \doteq \Omega \times(0, T)$ and $\Sigma \doteq \Gamma \times(0, T)$, for some given final reference time $T>0$. We will indicate with $u: \bar{Q} \rightarrow \mathbb{R}^{3}$ the displacement of the body from the reference configuration and prescribe some non-homogeneous Dirichlet boundary conditions. In particular, we assign the function $u^{\text {Dir }}: \bar{Q} \rightarrow \mathbb{R}^{3}$ and impose $u \equiv u^{\text {Dir }}$ on $\Sigma$.

In the following, we shall be using Korn's inequality (see, e.g., [21, Thm. 3.1, p. 110])

$$
\exists c_{\text {Korn }}>0: c_{\text {Korn }}\|u\|_{H^{1}\left(\Omega ; \mathbb{R}^{3}\right)}^{2} \leq\|u\|_{L^{2}\left(\Gamma ; \mathbb{R}^{3}\right)}^{2}+\|\varepsilon(u)\|_{L^{2}\left(\Omega ; \mathbb{R}_{\text {sym }}^{3 \times 3}\right)}^{2} \quad \forall u \in H^{1}\left(\Omega ; \mathbb{R}^{3}\right) .
$$

2.3. Constitutive relations. Within the small-deformation regime, we additively decompose the deformation

$$
\varepsilon(u)=\varepsilon^{\mathrm{el}}+\varepsilon^{\mathrm{tr}},
$$

into its elastic part $\varepsilon^{\mathrm{el}}$ and the inelastic (or transformation) part $\varepsilon^{\text {tr }}$.

Moreover, we introduce the additive decomposition of the total stress $\sigma^{\text {tot }}$ into a local part $\sigma$ and a nonlocal part $\sigma^{\text {nl }}$, namely

$$
\sigma^{\mathrm{tot}}=\sigma+\sigma^{\mathrm{nl}}
$$

We assume elastic material response and hence let the local part $\sigma$ of the stress fulfill

$$
L \sigma=\varepsilon^{\mathrm{el}},
$$

where $L$ is the compliance 4 -tensor $L \doteq C^{-1}$ with

$$
C \doteq 2 G\left(I_{4}-\frac{1}{3} I_{2} \otimes I_{2}\right)+K\left(I_{2} \otimes I_{2}\right) .
$$

Here $C$ is the elasticity tensor, $G>0$ is the shear modulus, $K>0$ is the bulk modulus, and $I_{k}$ is the identity $k$-tensor.

As for the nonlocal stress $\sigma^{\text {nl }}$, we let $(\mathrm{D} \varepsilon)_{i j k}=\varepsilon_{i j, k}$ and assume

$$
\left(\sigma^{\mathrm{nl}}\right)_{i j}=-\mu \varepsilon_{i j, k k}
$$


where $\mu$ is positive. This fourth-order contribution is assumed to give rise to some multiscale competition, nonlocal effect, or interfacial energy, and within the SMA context can be traced back at least to Falk [23]. We could of course accommodate some more generality by choosing $-\operatorname{div}(A \mathrm{D} \varepsilon)$ for some suitable positive definite 6-tensor $A$ instead of $-\mu \varepsilon_{i j, k k}$ (which corresponds to $\left.\mu I_{6}\right)$. That is, in components, $\left(\sigma^{\mathrm{nl}}\right)_{i j}=-(A \mathrm{D} \varepsilon)_{i j k, k}$. Note that some fourthorder contribution is also considered in $[16,17]$ where compactness for $\operatorname{tr} \varepsilon$ is exploited. For instance, the choice of [16] corresponds to $\left(\sigma^{\mathrm{nl}}\right)_{i j}=-\Delta(\operatorname{div} u) \delta_{i j}$, (Kronecker) or, taking into account the latter notation $A_{i j k \ell m n}=\delta_{i j} \delta_{\ell m} \delta_{k n}$. The latter choice is not admissible in our setting as $A$ is not positive definite.

Among other possible compactifying choices one could consider [1]

$$
\left(\sigma^{\mathrm{nl}}\right)_{i j}(x)=\int_{\Omega} K(x, y)\left(\varepsilon_{i j}(x)-\varepsilon_{i j}(y)\right) d y,
$$

for some suitable singular kernel $K$.

2.4. Inelastic evolution. Let us specify the Drucker-Prager yield function $F$ as

$$
F(\sigma) \doteq \varepsilon_{L}(|s|+3 \alpha p),
$$

written in terms of the standard decomposition

$$
p \doteq\left(I_{2}: \sigma\right) / 3, \quad s \doteq \sigma-p I_{2} .
$$

Here, $\varepsilon_{L}>0$ is a material constant representing a measure of the maximum strain obtainable through alignment of the martensite variants. For $\alpha=0$, the function $F$ reduces to a function of von Mises type and corresponds to the situation of equal transformation response in traction and compression. The asymmetric behavior of the material model can be modeled by choosing $\alpha \neq 0$ instead.

The yield function $F$ is generally defined up to a constant. On the other hand, $F$ is not a priori non-negative nor bounded below. Indeed, $F$ turns out to be bounded below for all uniaxial tension tests with small $\alpha$. As for hydrostatic tests, any negative value of $F$ is a priori reachable. We shall stress however that the model bears some consistency just within some confined pressure range. Namely, (although math is still consistent) the forthcoming transformation dynamics cannot describe the arbitrarily negative-pressure situation.

Concerning the inelastic part $\varepsilon^{\text {tr }}$ of the deformation we shall specify relation (1.1) in the current non-smooth setting by letting

$$
\varepsilon^{\operatorname{tr}} \in \xi \partial F(\sigma) .
$$

We recall that $\xi$ represents the volume fraction of single-variant martensite, and note that $F: \mathbb{R}_{\mathrm{sym}}^{3 \times 3} \rightarrow \mathbb{R}$ is convex and positively homogeneous. In particular, the symbol $\partial$ denotes the possibly set-valued subdifferential in the sense of Convex Analysis.

By combining the elastic response (2.3) and the inelastic ansatz (2.7), we finally obtain the constitutive relation of the material in the form

$$
L \sigma+\xi \partial F(\sigma) \ni \varepsilon .
$$

Before moving on, let us explicitly compute that

$$
\partial F(\sigma)=\varepsilon_{L}\left(\partial|s|+\alpha I_{2}\right) .
$$

Hence, owing to the above definitions and letting $\theta \doteq\left(I_{2}: \varepsilon\right) / 3$ and $e \doteq \varepsilon-\theta I_{2}$, one readily checks that relation (2.8) entails

$$
s \in 2 G\left(e-\varepsilon_{L} \xi \partial|s|\right), \quad p=3 K\left(\theta-\alpha \varepsilon_{L} \xi\right) .
$$


2.5. Evolution of $\boldsymbol{\xi}$. In the spirit of generalized plasticity models [36], we directly prescribe the evolution of the single-variant martensite volume fraction $\xi$. In particular, given the input $t \mapsto F(\sigma(t))$, we assume $t \mapsto \xi(t)$ to be given by solving

$$
\dot{\xi}+\partial I_{K(F(\sigma))}(\xi) \ni 0, \quad \xi(0)=\xi_{0}
$$

where the non-empty, closed, convex set $K$ is prescribed by means of two non-decreasing and Lipschitz continuous functions $\lambda_{\ell}, \lambda_{u}: \mathbb{R} \rightarrow[0,1]$ as

$$
K(F) \doteq\left[\lambda_{\ell}(F), \lambda_{u}(F)\right] \quad \forall F \geq 0 .
$$

Relation (2.9) is generally referred to as a generalized play operator $[15,30,56]$ or a sweeping process driven by $K[42,43,44]$. In particular, the evolution in (2.9) is rate-independent [56] and the input-output behavior for (2.9) is depicted in Figure 1. Note that horizontal paths $(\xi$ constant) are reversible whereas $\xi$ can just increase (decrease) along $\xi=\lambda_{\ell}(F(\sigma))\left(\xi=\lambda_{u}(F(\sigma))\right.$, respectively).

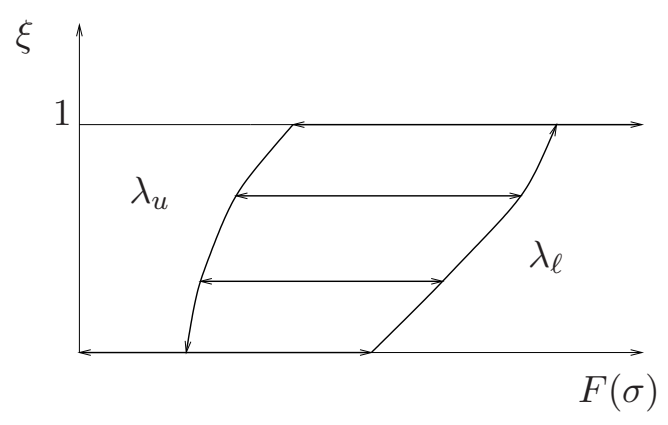

Figure 1. Diagram of the evolution of $\xi$

Owing to the specific form (2.6) of $F$ we readily check that one can rewrite the evolution problem (2.9) in terms of $\varepsilon=\varepsilon(u)$ only [10]. To this aim, we shall invert the constitutive relation (2.8). Indeed, we first observe that the compliance 4-tensor $L$ can be expressed as

$$
L=\frac{1}{2 G} \operatorname{dev}+\frac{1}{9 K} I_{2} \operatorname{tr},
$$

where dev is the projection of $\mathbb{R}_{\mathrm{sym}}^{3 \times 3}$ onto $\mathbb{R}_{\mathrm{dev}}^{3 \times 3}:=\left\{\eta \in \mathbb{R}_{\mathrm{sym}}^{3 \times 3}\right.$ such that $\left.\eta: I_{2}=0\right\}$ and tr is the trace operator given by $\operatorname{tr} \eta \doteq \eta: I_{2}$. Moreover, every element $\varphi \in \partial F(\sigma)$ admits the representation

$$
\varphi=\varepsilon_{L}\left(\eta+\alpha I_{2}\right),
$$

with

$$
\eta \begin{cases}\in B_{1}(0) \cap \mathbb{R}_{\operatorname{dev}}^{3 \times 3} & \text { if } \operatorname{dev} \sigma=0, \\ =\frac{\operatorname{dev} \sigma}{|\operatorname{dev} \sigma|} & \text { if } \operatorname{dev} \sigma \neq 0 .\end{cases}
$$

Then, the constitutive relation (2.8) is equivalent to

$$
\begin{aligned}
\operatorname{dev} \varepsilon & =\frac{1}{2 G} \operatorname{dev} \sigma+\varepsilon_{L} \xi \eta, \\
\operatorname{tr} \varepsilon & =\frac{1}{3 K} \operatorname{tr} \sigma+3 \varepsilon_{L} \alpha \xi I_{2} .
\end{aligned}
$$

As we have that $\eta: \operatorname{dev} \sigma=|\eta||\operatorname{dev} \sigma|$, we conclude for

$$
|\operatorname{dev} \varepsilon|=\frac{1}{2 G}|\operatorname{dev} \sigma|+\varepsilon_{L} \xi|\eta|,
$$


and the inverse relation reads

$$
|\operatorname{dev} \sigma|=2 G\left(|\operatorname{dev} \varepsilon|-\varepsilon_{L} \xi\right)^{+},
$$

where $(\cdot)^{+}=\max \{\cdot, 0\}$ is the usual positive part. The constitutive mapping $H:(\xi, \varepsilon) \mapsto \sigma$ can thus be written explicitly as

$$
\sigma=2 G\left(|\operatorname{dev} \varepsilon|-\varepsilon_{L} \xi\right)^{+} \frac{\operatorname{dev} \varepsilon}{|\operatorname{dev} \varepsilon|}+K\left(\operatorname{tr} \varepsilon-3 \varepsilon_{L} \alpha \xi\right) I_{2} .
$$

In particular, the mapping $\hat{F}=F \circ H$ has the form

$$
\hat{F}(\xi, \varepsilon)=\varepsilon_{L}\left(2 G\left(|\operatorname{dev} \varepsilon|-\varepsilon_{L} \xi\right)^{+}+3 K \alpha \operatorname{tr} \varepsilon-9 \varepsilon_{L} K \alpha^{2} \xi\right) .
$$

Now, let us define the functions $\hat{\lambda}_{l}(\varepsilon), \hat{\lambda}_{u}(\varepsilon)$ by letting

$$
\lambda_{i}(\widehat{F}(\xi, \varepsilon))=\xi \Leftrightarrow \xi=\widehat{\lambda}_{i}(\varepsilon) \text { for } i=\ell, u,
$$

Since $\hat{F}$ is decreasing in $\xi$, the mappings $\hat{\lambda}_{l}(\varepsilon), \hat{\lambda}_{u}(\varepsilon)$ turn out to be well defined. Introducing $\widehat{K}: \mathbb{R}_{\text {sym }}^{3 \times 3} \rightarrow 2^{[0,1]}$ by setting

$$
\widehat{K}(\varepsilon) \doteq\left[\widehat{\lambda}_{\ell}(\varepsilon), \widehat{\lambda}_{u}(\varepsilon)\right] \quad \forall \varepsilon \in \mathbb{R}_{\mathrm{sym}}^{3 \times 3},
$$

relation (2.9) finally turns out to be equivalent to

$$
\dot{\xi}+\partial I_{\widehat{K}(\varepsilon)}(\xi) \ni 0, \quad \xi(0)=\xi_{0} .
$$

We shall use this possible equivalent formulation for the evolution of $\xi$ in the following. Let us just stress that $\widehat{\lambda}_{\ell}, \widehat{\lambda}_{u}$ are Lipschitz continuous and that both $K$ and $\widehat{K}$ are Lipschitz continuous in the standard Hausdorff metric with respect to input variation. Recall that the Hausdorff distance between two non-empty sets $A, B \subset E, E$ being a normed set with norm $|\cdot|_{E}$, is given by

$$
d_{E}(A, B) \doteq \max \left\{\sup _{a \in A} \inf _{b \in B}|a-b|_{E} \sup _{b \in B} \inf _{a \in A}|a-b|_{E}\right\} .
$$

2.6. Associativity. The ATL model turns out to be associative in the standard plasticity sense [28]. The associative structure of the ATL model is revealed by defining the Gibbs energy density $G$ and the dissipation density $D$ as

$$
\begin{aligned}
G(\sigma, \xi) & \doteq-\frac{1}{2} \sigma: L \sigma-\xi F(\sigma)+h(\xi) \\
D(\sigma, \xi, \dot{\xi}) & \doteq \sup \left\{\dot{\xi} q: q \in-K(F(\sigma))-F(\sigma)+h^{\prime}(\xi)+\xi\right\},
\end{aligned}
$$

with $h$ given by

$$
h(\xi):=\int_{\xi_{0}}^{\xi} \lambda_{m}^{-1}(\xi) d \xi, \quad \xi_{0} \in\left(\inf \lambda_{\ell}, \sup \lambda_{u}\right),
$$

where $\lambda_{m}:=\left(\lambda_{\ell}+\lambda_{u}\right) / 2$ is the midline between $\lambda_{\ell}$ and $\lambda_{u}$, and $\lambda_{m}^{-1}$ is a fixed selection of the inverse (in the sense of maximal monotone graphs) of $\lambda_{m}$. Indeed, $D$ turns out to be the partial Legendre conjugate $I_{-K(F(\sigma))-F(\sigma)+h^{\prime}(\xi)+\xi}^{*}$ (with respect to $\dot{\xi}$ ) of the indicator function of the moving convex set $-K(F(\sigma))-F(\sigma)+h^{\prime}(\xi)+\xi$ and we readily compute that

$$
\begin{aligned}
\partial_{\sigma}(-G(\sigma, \xi)) & =L \sigma+\xi \partial F(\sigma)=\varepsilon, \\
-\partial_{\xi} G(\sigma, \xi) & =F(\sigma)-h^{\prime}(\xi), \\
\partial_{\dot{\xi}} D(\sigma, \xi, \dot{\xi}) & =\partial_{\dot{\xi}} I_{-K(F(\sigma))-F(\sigma)+h^{\prime}(\xi)+\xi}^{*}(\dot{\xi})
\end{aligned}
$$

with obvious notation for partial subdifferentials. Hence, the constitutive relation (2.8) and the flow rule (2.9) can be rewritten as the system

$$
\partial_{(\dot{\sigma}, \dot{\xi})} D(\sigma, \xi, \dot{\xi})+\partial_{(\sigma, \xi)}(-G(\sigma, \xi)) \ni\left(\begin{array}{l}
\varepsilon \\
0
\end{array}\right), \quad \xi(0)=\xi_{0} .
$$


An associative formulation for the ATL model in the variables $(\varepsilon, \xi)$ is available upon choosing the free energy density $\psi$ and the dissipation density $\widehat{D}$ as

$$
\begin{aligned}
\psi(\varepsilon, \xi) & \doteq \sup _{\sigma \in \mathbb{R}_{\mathrm{sym}}^{3 \times 3}}(\varepsilon: \sigma+G(\sigma, \xi)) \\
\widehat{D}(\varepsilon, \xi, \dot{\xi}) & \doteq \sup \left\{\dot{\xi} q: q \in-\widehat{K}(\varepsilon)+\widehat{F}(\xi, \varepsilon)-h^{\prime}(\xi)+\xi\right\} .
\end{aligned}
$$

In particular, by recalling that $-G$ is the partial Legendre conjugate of $\psi$ with respect to $\varepsilon$, we get

$$
\begin{aligned}
\partial_{\varepsilon} \psi(\varepsilon, \xi) & =\sigma, \\
\partial_{\xi} \psi(\varepsilon, \xi) & =\partial_{\xi} G(\sigma, \xi)=-F(\sigma)+h^{\prime}(\xi)=-\widehat{F}(\xi, \varepsilon)+h^{\prime}(\xi), \\
\partial_{\dot{\xi}} \widehat{D}(\varepsilon, \xi, \dot{\xi}) & =\partial_{\dot{\xi}} I_{-\widehat{K}(\varepsilon)+\widehat{F}(\xi, \varepsilon)-h^{\prime}(\xi)+\xi}(\dot{\xi}) .
\end{aligned}
$$

Hence, the constitutive relation (2.8) and the flow rule (2.11) turn out to be

$$
\partial_{(\dot{\varepsilon}, \dot{\xi})} \widehat{D}(\varepsilon, \xi, \dot{\xi})+\partial_{(\varepsilon, \xi)} \psi(\varepsilon, \xi) \ni\left(\begin{array}{c}
\sigma \\
0
\end{array}\right), \quad \xi(0)=\xi_{0} .
$$

Let us explicitly mention that, despite the associative variational structure of the ATL model, classical existence results for quasi-variational inequalities seem not directly applicable to the ATL model, the basic obstruction in this direction being the non-smoothness of the related functionals. On the other hand, at least for the zero-dimensional constitutive problem, some ad hoc analysis based on order methods in the spirit of [51] may be considered. We shall develop these considerations elsewhere.

Given the above introduced associative reformulations (2.12)-(2.13), the thermodynamic consistency of the ATL model can be readily checked. Indeed, for all suitably smooth evolutions, the Clausius-Duhem inequality holds in either one of the following equivalent forms

$$
\begin{aligned}
& \dot{\varepsilon}: \sigma-\frac{d}{d t}(\varepsilon: \sigma+G)=-\dot{G}-\varepsilon: \dot{\sigma}=\dot{\xi}\left(F(\sigma)-h^{\prime}(\xi)\right) \stackrel{(2.9)}{\geq} 0 \\
& \frac{d}{d t}(\psi-\varepsilon: \sigma)+\varepsilon: \dot{\sigma}=\dot{\psi}-\sigma: \dot{\varepsilon}=-\dot{\xi}\left(\widehat{F}(\xi, \varepsilon)-h^{\prime}(\xi)\right) \stackrel{(2.11)}{\leq} 0 .
\end{aligned}
$$

2.7. Quasistatic equilibrium. By coupling to the above-introduced constitutive relation (2.8) and the evolution law for $\xi$ in (2.9) with the quasistatic equilibrium system, we are lead to consider the problem of finding the displacement $u: \bar{Q} \rightarrow \mathbb{R}^{3}$, the (local) stress $\sigma: Q \rightarrow \mathbb{R}_{\mathrm{sym}}^{3 \times 3}$, and the single-variant martensitic volume fraction $\xi: Q \rightarrow[0,1]$ of the body such that

$$
\begin{aligned}
& \operatorname{div} \sigma^{\text {tot }}+b=0 \text { on } Q \text {, } \\
& u=u^{\text {Dir }} \text { on } \Sigma \text {, } \\
& \mu \varepsilon_{i j, k}(u) n_{k}=0 \text { on } \Sigma \text {, } \\
& L \sigma+\xi \partial F(\sigma) \ni \varepsilon \text { on } Q, \\
& \dot{\xi}+\partial I_{K(F(\sigma))}(\xi) \ni 0 \text { on } Q \text {, } \\
& \xi(0)=\xi_{0} \quad \text { on } \Omega \text {. }
\end{aligned}
$$

Here, $b$ is the body force density, $u^{\text {Dir }}$ is the given boundary displacement, $n$ is the outward normal to $\Gamma$, and $\xi_{0}: \Omega \rightarrow[0,1]$ is a given initial volume fraction of single-variant martensite.

\section{MAin EXistence Result}

The main issue of the paper is that of providing an existence result for the quasistatic evolution problem. In particular, we will focus on a variational formulation of the system in (2.14)-(2.19). 
In order to introduce the notion of weak solution we shall be dealing with, we multiply equation (2.14) by $v \in C_{\mathrm{c}}^{\infty}\left(\Omega ; \mathbb{R}^{3}\right)$ and integrate on $\Omega$ in order to get

$$
\int_{\Omega}\left(\sigma+\sigma^{\mathrm{nl}}\right): \varepsilon(v)=\int_{\Omega} b \cdot v
$$

By taking into account the boundary condition (2.16) we have

$$
\begin{aligned}
& \int_{\Omega} \sigma^{\mathrm{nl}}: \varepsilon(v)=-\int_{\Omega} \mu \varepsilon_{i j, k k}(u) \varepsilon_{i j}(v) \\
& =-\int_{\Gamma} \mu \varepsilon_{i j, k}(u) \varepsilon_{i j}(v) n_{k} d \Gamma+\int_{\Omega} \mu \varepsilon_{i j, k}(u) \varepsilon_{i j, k}(v)=\int_{\Omega} \mu \varepsilon_{i j, k}(u) \varepsilon_{i j, k}(v),
\end{aligned}
$$

and (3.1) yields

$$
\int_{\Omega}(\mu \mathrm{D} \varepsilon(u) \because \mathrm{D} \varepsilon(v)+\sigma: \varepsilon(v))=\int_{\Omega} b \cdot v
$$

Let us write

$$
u=w+u^{\text {Dir }},
$$

where we recall that the function $u^{\text {Dir }}$ is defined on the whole $\bar{Q}$, and introduce the spaces and the functional

$$
\begin{gathered}
W \doteq H^{1}\left(\Omega ; \mathbb{R}^{3}\right), \quad V \doteq\left\{w \in W: \varepsilon(w) \in H^{1}\left(\Omega ; \mathbb{R}_{\mathrm{sym}}^{3 \times 3}\right), w=0 \quad \text { on } \Gamma\right\} \\
\langle f, v\rangle:=\int_{\Omega} b \cdot v-\int_{\Omega} \mu \mathrm{D} \varepsilon\left(u^{\mathrm{Dir}}\right) \because \mathrm{D} \varepsilon(v) \quad \forall v \in V .
\end{gathered}
$$

We make the following assumptions on data

$$
\begin{aligned}
& b \in H^{1}\left(0, T ; L^{2}\left(\Omega ; \mathbb{R}^{3}\right)\right), \\
& u^{\text {Dir }} \in H^{1}(0, T ; W), \quad \varepsilon\left(u^{\text {Dir }}\right) \in H^{1}\left(0, T ; H^{1}\left(\Omega ; \mathbb{R}_{\text {sym }}^{3 \times 3}\right)\right) .
\end{aligned}
$$

Furthermore, we suppose that the initial data satisfy

$$
\begin{aligned}
& w^{0} \in V, \quad \sigma^{0} \in L^{2}\left(\Omega ; \mathbb{R}_{\mathrm{sym}}^{3 \times 3}\right), \quad \xi^{0} \in L^{2}(\Omega), \\
& \int_{\Omega}\left(\mu \mathrm{D} \varepsilon\left(w^{0}\right) \because \mathrm{D} \varepsilon(v)+\sigma^{0}: \varepsilon(v)\right)=\left\langle f_{0}, v\right\rangle \quad \forall v \in V, \\
& \xi^{0} \in K\left(F\left(\sigma^{0}\right)\right), \quad L \sigma^{0}+\xi^{0} \partial F\left(\sigma^{0}\right) \ni \varepsilon\left(w^{0}+u_{0}^{\text {Dir }}\right) \quad \text { a.e. in } \Omega,
\end{aligned}
$$

where $f_{0}=f(0)$ and $u_{0}^{\text {Dir }}=u^{\text {Dir }}(0)$. We shall be concerned in the following problem.

Definition 3.1 (Quasistatic Evolution Problem). To find

$$
w \in H^{1}(0, T ; V), \quad \sigma \in H^{1}\left(0, T ; L^{2}\left(\Omega ; \mathbb{R}_{\mathrm{sym}}^{3 \times 3}\right)\right), \quad \text { and } \xi \in H^{1}\left(0, T ; L^{2}(\Omega)\right)
$$

such that

$$
\begin{aligned}
& \int_{\Omega}(\mu \mathrm{D} \varepsilon(w) \because \mathrm{D} \varepsilon(v)+\sigma: \varepsilon(v))=\langle f, v\rangle \quad \forall v \in V, \text { a.e. in }(0, T), \\
& L \sigma+\xi \partial F(\sigma) \ni \varepsilon\left(w+u^{\mathrm{Dir}}\right) \quad \text { a.e. in } Q, \\
& \dot{\xi}+\partial I_{K(F(\sigma))}(\xi) \ni 0 \quad \text { a.e. in } Q, \\
& w(0)=w^{0} \quad \text { in } V, \quad \sigma(0)=\sigma^{0}, \quad \xi(0)=\xi^{0} \quad \text { a.e. in } \Omega .
\end{aligned}
$$

Our main result reads as follows.

Theorem 3.2 (Existence for the Quasistatic Evolution Problem). Let (3.2)-(3.6) hold. Then, the Quasistatic Evolution Problem admits a solution. 
The proof of Theorem 3.2 will be carried out in the remainder of the paper by means of a time-discretization argument. In particular, we will pass to the limit as the fineness of the time-partition goes to zero in a sequence of approximating trajectories which are obtained by solving an incremental step-problem. As the whole evolution is indeed rate-independent, the incremental problem turns out to bear a specific interest in itself and is here explicitly stated for the sake of definiteness.

Definition 3.3 (Incremental Problem). Given $\widehat{f} \in V^{*}, \widehat{\xi} \in L^{2}(\Omega)$ and $u^{\text {Dir }} \in W$, to find $w \in V$, $\sigma \in L^{2}\left(\Omega ; \mathbb{R}_{\mathrm{sym}}^{3 \times 3}\right)$, and $\xi \in L^{2}(\Omega)$ such that

$$
\begin{aligned}
& \int_{\Omega}(\mu \mathrm{D} \varepsilon(w) \because \mathrm{D} \varepsilon(v)+\sigma: \varepsilon(v))=\langle\widehat{f}, v\rangle \quad \forall v \in V, \\
& L \sigma+\xi \partial F(\sigma) \ni \varepsilon\left(w+u^{\mathrm{Dir}}\right) \quad \text { a.e. in } \Omega, \\
& (\xi-\widehat{\xi})+\partial I_{\widehat{K}\left(\varepsilon\left(w+u^{\mathrm{Dir}}\right)\right)}(\xi) \ni 0 \quad \text { a.e. in } \Omega .
\end{aligned}
$$

\section{Existence for the Incremental Problem}

In preparation of the proof of Theorem 3.2 we shall check for the following.

Theorem 4.1 (Existence for the Incremental Problem). The Incremental Problem has a solution.

The rest of this section is devoted to the proof of this result. We aim at applying Schauder's fixed-point theorem. Given $\widetilde{w} \in W$, from relation (3.13) we firstly determine

$$
\tilde{\xi}=\left(1+\partial I_{\widehat{K}\left(\varepsilon\left(\widetilde{w}+u^{\text {Dir }}\right)\right)}\right)^{-1}(\widehat{\xi}),
$$

namely, $\tilde{\xi}$ is almost everywhere the pointwise projection of $\widehat{\xi}$ onto the interval $\widehat{K}\left(\varepsilon\left(\widetilde{w}+u^{\text {Dir }}\right)\right)$. Secondly we consider the following sub-problem.

Definition 4.2 (Equilibrium Incremental Problem). Given $\tilde{f} \in V^{*}, \tilde{\xi} \in L^{2}(\Omega)$ and $u^{\text {Dir }} \in W$, to find $w \in V$ and $\sigma \in L^{2}\left(\Omega ; \mathbb{R}_{\mathrm{sym}}^{3 \times 3}\right)$ such that

$$
\begin{aligned}
\int_{\Omega}(\mu \mathrm{D} \varepsilon(w) \because \mathrm{D} \varepsilon(v)+\sigma: \varepsilon(v)) & =\langle\tilde{f}, v\rangle \quad \forall v \in V, \\
L \sigma+\tilde{\xi} \partial F(\sigma) & \ni \varepsilon\left(w+u^{\mathrm{Dir}}\right) \text { a.e. in } \Omega .
\end{aligned}
$$

The Equilibrium Incremental Problem will be shown to possess a unique solution. The map $\widetilde{w} \rightarrow w$, where $w$ is the solution of the Equilibrium Incremental Problem, will turn out to have a fixed point, which in turn solves the Incremental Problem.

We start from the following.

Lemma 4.3 (Well-posedness of the Equilibrium Incremental Problem). The Equilibrium Incremental Problem has a unique solution.

Proof. We first prove existence. Let us introduce the function $\mathcal{W}^{*}:[0,1] \times \mathbb{R}_{\mathrm{sym}}^{3 \times 3} \rightarrow \mathbb{R}$ as

$$
\mathcal{W}^{*}(\xi, \sigma) \doteq \frac{1}{2} \sigma: L \sigma+\xi F(\sigma)-\varepsilon\left(u^{\text {Dir }}\right): \sigma
$$

and its partial Legendre conjugate with respect to $\sigma$

$$
\mathcal{W}(\xi, \varepsilon)=\sup _{\sigma \in \mathbb{R}_{\mathrm{sym}}^{3 \times 3}}\left(\varepsilon: \sigma-\mathcal{W}^{*}(\xi, \sigma)\right) .
$$

This allows to rewrite (4.2) as

$$
\varepsilon(w) \in \partial_{\sigma} \mathcal{W}^{*}(\tilde{\xi}, \sigma) \quad \text { or } \quad \sigma \in \partial_{\varepsilon} \mathcal{W}(\tilde{\xi}, \varepsilon(w)) \quad \text { a.e. in } \Omega .
$$


The incremental equilibrium problem (4.1)-(4.2) is equivalent to the following minimization problem

$$
\min _{w \in V} I(w)
$$

for the functional $I: W \rightarrow \mathbb{R}$ defined by

$$
I(w) \doteq \int_{\Omega}\left(\frac{\mu}{2}|\mathrm{D} \varepsilon(w)|^{2}+\mathcal{W}(\tilde{\xi}, \varepsilon(w))\right)-\langle\tilde{f}, w\rangle .
$$

Henceforth $c, c^{\prime}$ will stand for any positive constant, possibly depending on data and varying from line to line.

It is now easy to see that problem (4.3) has a solution since $I$ is continuous and coercive in $V$. Indeed, we readily check that

$$
\mathcal{W}^{*}(\tilde{\xi}, \sigma) \leq c\left(1+|\sigma|^{2}\right) \quad \forall \sigma \in \mathbb{R}_{\mathrm{sym}}^{3 \times 3} .
$$

Hence, we can write

$$
\mathcal{W}(\tilde{\xi}, \varepsilon) \geq \sup _{\sigma \in \mathbb{R}_{\text {sym }}^{333}}\left(\varepsilon: \sigma-c\left(1+|\sigma|^{2}\right)\right)=\sup _{z>0}\left(z|\varepsilon|-c-c z^{2}\right)=\frac{|\varepsilon|^{2}}{4 c}-c .
$$

By means of Korn's inequality (2.1) we therefore have

$$
\begin{aligned}
& I(w) \geq c\|\varepsilon(w)\|_{W}^{2}-\|\widetilde{f}\|_{V^{*}}\|w\|_{V}-c \\
& \geq \frac{c}{2}\|\varepsilon(w)\|_{W}^{2}+\frac{c}{2} c_{\text {Korn }}\|w\|_{W}^{2}-\|\widetilde{f}\|_{V^{*}}\|w\|_{V}-c \\
& \geq c\|w\|_{V}^{2}-\|\widetilde{f}\|_{V^{*}}\|w\|_{V}-c,
\end{aligned}
$$

and coercivity follows.

In order to prove the uniqueness for the Equilibrium Incremental Problem, let $w_{1}, w_{2} \in V$ be two solutions and check that

$$
\sigma_{1}=\sigma_{2}, \quad \mathrm{D} \varepsilon\left(w_{1}-w_{2}\right)=0 \text { a.e. in } \Omega .
$$

Indeed, by writing problem (4.1)-(4.2) for two solutions $w_{1}, \sigma_{1}$ and $w_{2}, \sigma_{2}$, taking the difference of the two equations (4.1) and testing by $w_{1}-w_{2}$, we get

$$
\int_{\Omega}\left(\mu\left|\mathrm{D} \varepsilon\left(w_{1}-w_{2}\right)\right|^{2}+\left(\sigma_{1}-\sigma_{2}\right): \varepsilon\left(w_{1}-w_{2}\right)\right)=0 .
$$

On the other hand, by the monotonicity of $\partial F$ we can write

$$
\begin{aligned}
& \left(\left(\varepsilon\left(w_{1}\right)-L \sigma_{1}\right)-\left(\varepsilon\left(w_{2}\right)-L \sigma_{2}\right)\right):\left(\sigma_{1}-\sigma_{2}\right) \\
& =\left(\varepsilon\left(w_{1}-w_{2}\right)-L\left(\sigma_{1}-\sigma_{2}\right)\right):\left(\sigma_{1}-\sigma_{2}\right) \geq 0,
\end{aligned}
$$

and hence

$$
\left(\sigma_{1}-\sigma_{2}\right): \varepsilon\left(w_{1}-w_{2}\right) \geq L\left(\sigma_{1}-\sigma_{2}\right):\left(\sigma_{1}-\sigma_{2}\right) \geq c\left|\sigma_{1}-\sigma_{2}\right|^{2} \geq 0 .
$$

We hence have the equalities (4.4) and the boundary condition $w=0$ on $\Gamma$ entails $w_{1}=w_{2}$. Indeed, by setting $\widetilde{w}=w_{1}-w_{2}$ and integrating $\varepsilon_{i k}(\widetilde{w})$ we get

$$
\int_{\Omega} \varepsilon_{i k}(\widetilde{w})=\frac{1}{2} \int_{\Omega}\left(\frac{\partial \widetilde{w}_{i}}{\partial x_{k}}+\frac{\partial \widetilde{w}_{k}}{\partial x_{i}}\right)=\frac{1}{2} \int_{\Gamma}\left(\widetilde{w}_{i} n_{k}+\widetilde{w}_{k} n_{i}\right)=0
$$

Hence, by Poincaré's inequality and the fact that $\mathrm{D} \varepsilon(\widetilde{w})=0$ we have that $\varepsilon(\widetilde{w})=0$. From Korn's inequality $(2.1)$ we finally deduce $\widetilde{w}=0$.

We now turn to the proof of Theorem 4.1. Let us introduce the set $M \subset W$ defined by

$$
M \doteq\left\{w \in V: \int_{\Omega}\left(|\mathrm{D} \varepsilon(w)|^{2}+|\varepsilon(w)|^{2}\right) \leq c_{0}, \quad w=0 \quad \text { on } \Gamma\right\},
$$


where $c_{0}$ is a positive constant to be defined later and introduce the operator $S: M \rightarrow V \subset W$, defined as the composition $S=S_{2} \circ S_{1}$, where $S_{1}: M \rightarrow L^{2}(\Omega)$ is given by

$$
S_{1}(\widetilde{w}) \doteq\left(1+\partial I_{\widehat{K}\left(\varepsilon\left(\widetilde{w}+u^{\text {Dir }}\right)\right)}\right)^{-1}(\widehat{\xi}),
$$

for every $\widetilde{w} \in M$. Note that the nonlinear operator $S_{1}$ is nothing but the pointwise projection of $\widehat{\xi}$ on the closed interval $\widehat{K}\left(\varepsilon\left(\widetilde{w}+u^{\text {Dir }}\right)\right)$. It is easy to check that, due to the Lipschitz continuity of the map $\widehat{F} \rightarrow \widehat{K}(\widehat{F})$, the operator $S_{1}$ fulfills

$$
\left\|S_{1}\left(\widetilde{w}_{1}\right)-S_{1}\left(\widetilde{w}_{2}\right)\right\|_{L^{2}(\Omega)} \leq c\left\|\varepsilon\left(\widetilde{w}_{1}-\widetilde{w}_{2}\right)\right\|_{L^{2}\left(\Omega ; \mathbb{R}_{\mathrm{sym}}^{3 \times 3}\right)},
$$

and it is hence globally Lipschitz continuous from $W$ to $L^{2}(\Omega)$. Moreover $S_{2}: L^{2}(\Omega) \rightarrow V \subset W$ is defined to be such that $S_{2}(\widetilde{\xi})$ is the unique solution $w \in V \subset W$ to the Equilibrium Incremental Problem with data $\widetilde{f}=\widehat{f} \in V^{*}, \widetilde{\xi}=\widehat{\xi} \in L^{2}(\Omega)$, and $u^{\text {Dir }} \in W$.

The set $M$ is clearly convex and closed in $W$. It is moreover easy to verify that it is also compact in $W$. For the sake of applying Schauder's theorem [13], we need to check that, for $c_{0}$ sufficiently large, one has that $S(M) \subset M, \overline{S(M)}$ (closure in $W$ ) is compact in $W$, and $S: W \rightarrow W$ is continuous.

Take $\widetilde{w} \in M$. Then $w \doteq S(\widetilde{w})$ and $\widetilde{\xi} \doteq S_{1}(\widetilde{w})$ satisfy

$$
\begin{aligned}
& \int_{\Omega}\left(\mu|\mathrm{D} \varepsilon(w)|^{2}+\sigma: \varepsilon(w)\right)=\langle\widehat{f}, w\rangle, \\
& \sigma+\widetilde{\xi} C \partial F(\sigma) \ni C \varepsilon\left(w+u^{\text {Dir }}\right), \quad \text { a.e in } \Omega,
\end{aligned}
$$

along with a stress $\sigma \in L^{2}\left(\Omega ; \mathbb{R}_{\text {sym }}^{3 \times 3}\right)$. Hence, we have that

$$
\sigma=C \varepsilon\left(w+u^{\text {Dir }}\right)-\widetilde{\xi} C \eta,
$$

for a selection $\eta$ such that $\eta \in \partial F(\sigma)$ almost everywhere in $\Omega$ and

$$
\begin{aligned}
& \sigma: \varepsilon(w)=C \varepsilon\left(w+u^{\text {Dir }}\right): \varepsilon(w)-\widetilde{\xi} C \eta: \varepsilon(w) \\
& \geq c|\varepsilon(w)|^{2}-\frac{1}{c},
\end{aligned}
$$

where we have used the fact that $\partial F$ is bounded and $\widetilde{\xi} \in[0,1]$. Hence, from (4.7) and (4.8) we get

$$
\int_{\Omega}\left(|\mathrm{D} \varepsilon(w)|^{2}+|\varepsilon(w)|^{2}\right) \leq c^{\prime}
$$

If we now chose $c_{0} \doteq c^{\prime}$ we obtain that $S(M) \subset M$.

The compactness of $\overline{S(M)}$ in $W$ is now an immediate consequence of the inclusion $S(M) \subset M$ and of the compactness of $M$ in $W$.

It remains to prove the continuity of $S$ in the strong topology of $W$. To this aim, let $\widetilde{w}_{n} \rightarrow \widetilde{w}$ in $W$ and set $\widetilde{\xi}_{n}=S_{1}\left(\widetilde{w}_{n}\right)$. Due to $(4.6)$, we have $\widetilde{\xi}_{n} \rightarrow \widetilde{\xi}$, strongly in $L^{2}(\Omega)$, where $\widetilde{\xi} \doteq S_{1}(\widetilde{w})$. On the other hand, setting $w_{n}=S\left(\widetilde{w}_{n}\right)$, from estimate $(4.9)$ we have $\left\|\varepsilon\left(w_{n}\right)\right\|_{H^{1}\left(\Omega ; \mathbb{R}_{\mathrm{sym}}^{3 \times 3}\right)} \leq c$, which implies that there exists a not relabeled subsequence and an element $w \in V$ such that

$$
\varepsilon\left(w_{n}\right) \rightarrow \varepsilon(w) \quad \text { strongly in } L^{2}\left(\Omega ; \mathbb{R}_{\mathrm{sym}}^{3 \times 3}\right) \quad \text { and weakly in } H^{1}\left(\Omega ; \mathbb{R}_{\mathrm{sym}}^{3 \times 3}\right) .
$$

We now have to check that indeed the latter convergence holds for the whole sequence and that $w=S(\widetilde{w})$. Since $w_{n}$, together with $\sigma_{n}$, solves the Equilibrium Incremental Problem with data $\widehat{f}$ and $\widetilde{\xi}_{n}$, we obtain

$$
\begin{gathered}
\int_{\Omega} \mu\left|\mathrm{D} \varepsilon\left(w_{n}-w_{m}\right)\right|^{2}+\int_{\Omega}\left(\sigma_{n}-\sigma_{m}\right): \varepsilon\left(w_{n}-w_{m}\right)=0, \\
L\left(\sigma_{n}-\sigma_{m}\right)+\widetilde{\xi}_{n}\left(\eta_{n}-\eta_{m}\right)=\varepsilon\left(w_{n}-w_{m}\right)-\eta_{m}\left(\widetilde{\xi}_{n}-\widetilde{\xi}_{m}\right) \text { a.e. in } \Omega,
\end{gathered}
$$


for some $\eta_{i} \in \partial F\left(\sigma_{i}\right)$ almost everywhere in $\Omega$, for $i=n, m$. Now, from (4.12), by using the monotonicity and the boundedness of $\partial F$, we get

$$
c\left|\sigma_{n}-\sigma_{m}\right|^{2} \leq \varepsilon\left(w_{n}-w_{m}\right):\left(\sigma_{n}-\sigma_{m}\right)+\frac{1}{c}\left|\widetilde{\xi}_{n}-\widetilde{\xi}_{m}\right|^{2},
$$

and hence, (4.11) yields

$$
\int_{\Omega} \mu\left|\mathrm{D} \varepsilon\left(w_{n}-w_{m}\right)\right|^{2}+c \int_{\Omega}\left|\sigma_{n}-\sigma_{m}\right|^{2} \leq \frac{1}{c} \int_{\Omega}\left|\widetilde{\xi}_{n}-\widetilde{\xi}_{m}\right|^{2} .
$$

From this last estimate we obtain that $\mathrm{D} \varepsilon\left(w_{n}\right)$ and $\sigma_{n}$ are Cauchy sequences in $L^{2}\left(\Omega ; \mathbb{R}^{3 \times 3 \times 3}\right)$ and $L^{2}\left(\Omega ; \mathbb{R}_{\mathrm{sym}}^{3 \times 3}\right)$, respectively. By $(4.10)$, we have that $\mathrm{D} \varepsilon\left(w_{n}\right) \rightarrow \mathrm{D} \varepsilon(w)$ strongly in $L^{2}\left(\Omega ; \mathbb{R}^{3 \times 3 \times 3}\right)$. Moreover, there exists $\sigma \in L^{2}\left(\Omega ; \mathbb{R}_{\mathrm{sym}}^{3 \times 3}\right)$ such that $\sigma_{n} \rightarrow \sigma$ strongly in $L^{2}\left(\Omega ; \mathbb{R}_{\mathrm{sym}}^{3 \times 3}\right)$. It is now immediate to pass to the limit in (4.1), written for $w_{n}, \sigma_{n}$ and with datum $\widehat{f}$.

Next, by extracting a further (non-relabeled) subsequence, we can also assume the pointwise convergences $\widetilde{\xi}_{n} \rightarrow \widetilde{\xi}, \sigma_{n} \rightarrow \sigma$ and $\varepsilon\left(w_{n}\right) \rightarrow \varepsilon(w)$ a.e. in $\Omega$ and this allows to pass to the limit also in (4.2), written for $w_{n}, \sigma_{n}$ and $\widetilde{\xi}_{n}$. Hence, we conclude that $w$ and $\sigma$ solve

$$
\begin{aligned}
\int_{\Omega}(\mu \mathrm{D} \varepsilon(w) \because \mathrm{D} \varepsilon(v)+\sigma: \varepsilon(v)) & =\langle\widehat{f}, v\rangle \quad \forall v \in V, \\
L \sigma+\tilde{\xi} \partial F(\sigma) & \ni \varepsilon\left(w+u^{\text {Dir }}\right) \quad \text { a.e. in } \Omega,
\end{aligned}
$$

namely $w=S(\widetilde{w})$. Finally, the convergence for the whole sequence follows from the uniqueness of the solution of the Equilibrium Incremental Problem (4.1)-(4.2) (see Lemma 4.3).

\section{Proof of Theorem 3.2}

We shall make use of a time-discretization scheme. Let us introduce the uniform partition $\left\{0=t_{0}<t_{1}<\cdots<t_{N-1}<t_{N}=T\right\}$ with $t_{i} \doteq i \tau$, for $i=0, \ldots, N$, where $\tau$ is the diameter and $N \tau=T$. Letting $\left\{z_{i}\right\}_{i=0}^{N}$ be a vector, we denote by $z_{\tau}$ and $\bar{z}_{\tau}$ two functions of the time interval $[0, T]$ which interpolate the values of the vector $\left\{z_{i}\right\}$ piecewise linearly and backward constantly on the partition, respectively. Namely,

$$
\begin{gathered}
z_{\tau}(0) \doteq z_{0}, \quad z_{\tau}(t) \doteq \gamma_{i}(t) z_{i}+\left(1-\gamma_{i}(t)\right) z_{i-1} \\
\bar{z}_{\tau}(0) \doteq z_{0}, \quad \bar{z}_{\tau}(t) \doteq z_{i}, \quad \text { for } t \in((i-1) \tau, i \tau], \quad i=1, \ldots, N
\end{gathered}
$$

where

$$
\gamma_{i}(t) \doteq(t-(i-1) \tau) / \tau \quad \text { for } t \in((i-1) \tau, i \tau], \quad i=1, \ldots, N .
$$

Setting $f_{i} \doteq f\left(t_{i}\right)$ and $u_{i}^{\text {Dir }} \doteq u^{\operatorname{Dir}}\left(t_{i}\right)$, we now inductively construct a discrete solution to the Incremental Problem. Namely, we obtain $\left\{w_{i}\right\}_{i=0}^{N} \in V^{N+1},\left\{\sigma_{i}\right\}_{i=0}^{N} \in\left(L^{2}\left(\Omega ; \mathbb{R}_{\mathrm{sym}}^{3 \times 3}\right)\right)^{N+1}$, and $\left\{\xi_{i}\right\}_{i=0}^{N} \in\left(L^{2}(\Omega)\right)^{N+1}$ such that

$$
\begin{gathered}
w_{0}=w^{0} \text { in } V, \quad \sigma_{0}=\sigma^{0}, \quad \xi_{0}=\xi^{0} \quad \text { a.e. in } \Omega, \\
\int_{\Omega}\left(\mu \mathrm{D} \varepsilon\left(w_{i}\right) \because \mathrm{D} \varepsilon(v)+\sigma_{i}: \varepsilon(v)\right)=\left\langle f_{i}, v\right\rangle \quad \forall v \in V, \text { for } i=1, \ldots, N, \\
L \sigma_{i}+\xi_{i} \partial F\left(\sigma_{i}\right) \ni \varepsilon\left(w_{i}+u_{i}^{D}\right) \quad \text { a.e. in } \Omega, \text { for } i=1, \ldots, N, \\
\xi_{i}-\xi_{i-1}+\partial I_{K\left(F\left(\sigma_{i}\right)\right)}\left(\xi_{i}\right) \ni 0 \quad \text { a.e. in } \Omega, \text { for } i=1, \ldots, N .
\end{gathered}
$$


5.1. A priori estimates. Since $w_{i}$ satisfies (4.9) for $i=1, \ldots, N$ (the constant on the right side of (4.9) being independent of $\xi_{i}$ ), we have that

$$
\left\|w_{\tau}\right\|_{L^{\infty}(0, T ; V)} \leq c
$$

where here and henceforth all constants $c$ are independent of $\tau$. Taking the difference in (5.2)(5.3) for two consecutive time-steps we obtain that

$$
\begin{gathered}
\int_{\Omega} \mu\left|\mathrm{D} \varepsilon\left(w_{i}-w_{i-1}\right)\right|^{2}+\int_{\Omega}\left(\sigma_{i}-\sigma_{i-1}\right): \varepsilon\left(w_{i}-w_{i-1}\right)=\left\langle f_{i}-f_{i-1}, w_{i}-w_{i-1}\right\rangle \\
L\left(\sigma_{i}-\sigma_{i-1}\right)+\xi_{i-1}\left(\eta_{i}-\eta_{i-1}\right)+\left(\xi_{i}-\xi_{i-1}\right) \eta_{i}-\varepsilon\left(u_{i}^{\text {Dir }}-u_{i-1}^{\text {Dir }}\right) \\
=\varepsilon\left(w_{i}-w_{i-1}\right) \quad \text { a.e. in } \Omega
\end{gathered}
$$

for $\eta_{j} \in \partial F\left(\sigma_{j}\right)$ almost everywhere, $j=i, i-1$, and $i=1, \ldots, N$. If now we multiply equation (5.6) by $\left(\sigma_{i}-\sigma_{i-1}\right)$, we have to consider the term $\left(\xi_{i}-\xi_{i-1}\right) \eta_{i}\left(\sigma_{i}-\sigma_{i-1}\right)$. We aim to show that the latter is almost everywhere non-negative. Indeed, wherever $\xi_{i}>\xi_{i-1}$ then $F\left(\sigma_{i}\right)>F\left(\sigma_{i-1}\right)$ and

$$
\left(\xi_{i}-\xi_{i-1}\right) \eta_{i}\left(\sigma_{i}-\sigma_{i-1}\right) \geq\left(\xi_{i}-\xi_{i-1}\right)\left(F\left(\sigma_{i}\right)-F\left(\sigma_{i-1}\right)\right) \geq 0 .
$$

An analogous trick applies to the points in $\Omega$ where $\xi_{i}<\xi_{i-1}$. Hence, we have

$$
\begin{aligned}
& L\left(\sigma_{i}-\sigma_{i-1}\right):\left(\sigma_{i}-\sigma_{i-1}\right) \leq \varepsilon\left(w_{i}-w_{i-1}\right):\left(\sigma_{i}-\sigma_{i-1}\right) \\
& +\varepsilon\left(u_{i}^{\mathrm{Dir}}-u_{i-1}^{\mathrm{Dir}}\right):\left(\sigma_{i}-\sigma_{i-1}\right)
\end{aligned}
$$

and, recalling (5.5),

$$
\begin{aligned}
& \int_{\Omega}\left(\mu\left|\mathrm{D} \varepsilon\left(w_{i}-w_{i-1}\right)\right|^{2}+L\left(\sigma_{i}-\sigma_{i-1}\right):\left(\sigma_{i}-\sigma_{i-1}\right)\right) \\
\leq & \left\langle f_{i}-f_{i-1}, w_{i}-w_{i-1}\right\rangle+\int_{\Omega} \varepsilon\left(u_{i}^{\text {Dir }}-u_{i-1}^{\text {Dir }}\right):\left(\sigma_{i}-\sigma_{i-1}\right) .
\end{aligned}
$$

Now, for every $w \in V$, we have (see (4.5)) $\int_{\Omega} \varepsilon(w)=0$, and therefore, by means of Poincaré's

$$
\left\|\varepsilon\left(w_{i}-w_{i-1}\right)\right\|_{L^{2}\left(\Omega ; \mathbb{R}_{\mathrm{sym}}^{3 \times 3}\right)} \leq c\left\|\mathrm{D} \varepsilon\left(w_{i}-w_{i-1}\right)\right\|_{L^{2}\left(\Omega ; \mathbb{R}^{3 \times 3 \times 3}\right)}
$$

and Korn's inequality (2.1), we get

$$
\left\|w_{i}-w_{i-1}\right\|_{V} \leq c\left\|\mathrm{D} \varepsilon\left(w_{i}-w_{i-1}\right)\right\|_{L^{2}\left(\Omega ; \mathbb{R}^{3 \times 3 \times 3}\right)} .
$$

Moreover, due to to (5.4) we readily check that

$$
\left|\xi_{i}-\xi_{i-1}\right|=\inf _{x \in K\left(F\left(\sigma_{i}\right)\right)}\left|x-\xi_{i-1}\right| \leq c\left|\sigma_{i}-\sigma_{i-1}\right| .
$$

Therefore, by using (5.9) and (5.10), from (5.8) we get

$$
\begin{aligned}
& \left\|w_{i}-w_{i-1}\right\|_{V}^{2}+\left\|\xi_{i}-\xi_{i-1}\right\|_{L^{2}(\Omega)}^{2}+\left\|\sigma_{i}-\sigma_{i-1}\right\|_{L^{2}\left(\Omega ; \mathbb{R}_{\mathrm{sym}}^{3 \times 3}\right)}^{2} \\
& \leq c\left(\left\|f_{i}-f_{i-1}\right\|_{V^{*}}^{2}+\left\|\varepsilon\left(u_{i}^{\mathrm{Dir}}-u_{i-1}^{\mathrm{Dir}}\right)\right\|_{L^{2}\left(\Omega ; \mathbb{R}_{\mathrm{sym}}^{3 \times 3}\right)}^{2 \times}\right) .
\end{aligned}
$$

Now, dividing this last estimate by $\tau$ and summing for $i=1$ to $N$, we immediately get

$$
\begin{aligned}
& \left\|w_{\tau}^{\prime}\right\|_{L^{2}(0, T ; V)}^{2}+\left\|\xi_{\tau}^{\prime}\right\|_{L^{2}\left(0, T ; L^{2}(\Omega)\right)}^{2}+\left\|\sigma_{\tau}^{\prime}\right\|_{L^{2}\left(0, T ; L^{2}\left(\Omega ; \mathbb{R}_{\mathrm{sym}}^{3 \times 3}\right)\right)}^{2} \\
& \leq c\left(\left\|f_{\tau}^{\prime}\right\|_{L^{2}\left(0, T ; V^{*}\right)}^{2}+\left\|\varepsilon\left(\partial_{t} u_{\tau}^{\mathrm{Dir}}\right)\right\|_{L^{2}\left(0, T ; L^{2}\left(\Omega ; \mathbb{R}_{\mathrm{sym}}^{3 \times 3}\right)\right)}^{2 \times 3}\right) .
\end{aligned}
$$

An easy calculation yields

$$
\begin{aligned}
& \left\|f_{\tau}^{\prime}\right\|_{L^{2}\left(0, T ; V^{*}\right)} \leq\left\|f^{\prime}\right\|_{L^{2}\left(0, T ; V^{*}\right)}, \\
& \left\|\varepsilon\left(\partial_{t} u_{\tau}^{\text {Dir }}\right)\right\|_{L^{2}\left(0, T ; L^{2}\left(\Omega ; \mathbb{R}_{\mathrm{sym}}^{3 \times 3}\right)\right)} \leq\left\|\varepsilon\left(\partial_{t} u^{\mathrm{Dir}}\right)\right\|_{L^{2}\left(0, T ; L^{2}\left(\Omega ; \mathbb{R}_{\mathrm{sym}}^{3 \times 3}\right)\right)},
\end{aligned}
$$


for every $\tau$. In particular, we have that

$$
\begin{aligned}
& f_{\tau} \rightarrow f \text { strongly in } H^{1}\left(0, T ; V^{*}\right), \\
& \varepsilon\left(\partial_{t} u_{\tau}^{\text {Dir }}\right) \rightarrow \varepsilon\left(\partial_{t} u^{\text {Dir }}\right) \text { strongly in } L^{2}\left(0, T ; L^{2}\left(\Omega ; \mathbb{R}_{\mathrm{sym}}^{3 \times 3}\right)\right),
\end{aligned}
$$

as $\tau \rightarrow 0$. Now, since $\xi_{i} \in[0,1]$ almost everywhere in $\Omega$ and

$$
\sigma_{i}=C \varepsilon\left(w_{i}+u_{i}^{\text {Dir }}\right)-\xi_{i} C \eta_{i} \quad \text { a.e. in } \Omega,
$$

for $i=1, \cdots, N$, where $\eta_{i} \in \partial F\left(\sigma_{i}\right)$ almost everywhere in $\Omega$, then it is easy to see that we have

$$
\left\|\xi_{\tau}\right\|_{L^{\infty}(Q)} \leq c, \quad\left\|\sigma_{\tau}\right\|_{L^{\infty}\left(0, T ; L^{2}\left(\Omega ; \mathbb{R}_{\mathrm{sym}}^{3 \times 3}\right)\right)} \leq c .
$$

Eventually, by combining all the above estimates, we get

$$
\left\|w_{\tau}\right\|_{H^{1}(0, T ; V)}+\left\|\xi_{\tau}\right\|_{H^{1}\left(0, T ; L^{2}(\Omega)\right)}+\left\|\sigma_{\tau}\right\|_{H^{1}\left(0, T ; L^{2}\left(\Omega ; \mathbb{R}_{\mathrm{sym}}^{3 \times 3}\right)\right)} \leq c .
$$

5.2. Extraction of strongly converging subsequences. From estimate (5.11) we deduce that there exists $w \in H^{1}(0, T ; V)$ such that, up to a not relabeled subsequence, we have

$$
w_{\tau} \rightarrow w \text { weakly in } H^{1}(0, T ; V) .
$$

By exploiting the compact embedding $H^{1}(0, T ; V) \subset C([0, T] ; W)$ we have

$$
\begin{aligned}
& \varepsilon\left(w_{\tau}\right) \rightarrow \varepsilon(w) \quad \text { strongly in } C\left([0, T] ; L^{2}\left(\Omega ; \mathbb{R}_{\mathrm{sym}}^{3 \times 3}\right)\right), \\
& \varepsilon\left(\bar{w}_{\tau}\right) \rightarrow \varepsilon(w) \quad \text { strongly in } L^{\infty}\left(0, T ; L^{2}\left(\Omega ; \mathbb{R}_{\mathrm{sym}}^{3 \times 3}\right)\right) .
\end{aligned}
$$

We now show that there exists $\xi \in H^{1}\left(0, T ; L^{2}(\Omega)\right)$ such that, up to some not relabeled subsequence, we have

$$
\xi_{\tau} \rightarrow \xi \quad \text { strongly in } C\left([0, T] ; L^{2}(\Omega)\right) .
$$

Indeed, (5.11) yields the existence of a $\xi \in H^{1}\left(0, T ; L^{2}(\Omega)\right)$ such that $\xi_{\tau} \rightarrow \xi$ weakly in $H^{1}\left(0, T ; L^{2}(\Omega)\right)$ and (5.13) follows immediately once we prove that $\xi_{\tau}$ is a Cauchy sequence in $C\left([0, T] ; L^{2}(\Omega)\right)$. Let us first introduce some notation by defining

$$
\mathcal{K}_{\tau} \doteq \widehat{K}\left(\varepsilon\left(\bar{w}_{\tau}+\bar{u}_{\tau}^{D}\right)\right) .
$$

Now relation (5.4) can be rewritten in the more compact form

$$
\xi_{\tau}^{\prime}+\partial I_{\mathcal{K}_{\tau}}\left(\bar{\xi}_{\tau}\right) \ni 0 \text { a.e. in } Q .
$$

In particular we have that

$$
\xi_{\tau}^{\prime}\left(\xi_{\tau}-v_{\tau}\right)=\xi_{\tau}^{\prime}\left(\bar{\xi}_{\tau}-v_{\tau}\right)+\xi_{\tau}^{\prime}\left(\xi_{\tau}-\bar{\xi}_{\tau}\right) \leq 0 \quad \text { a.e. in } Q, \quad \forall v_{\tau} \in \mathcal{K}_{\tau} \text { a.e. in } Q \text {. }
$$

Hence, we get, for every $\delta, \tau>0$,

$$
\xi_{\tau}^{\prime}\left(\xi_{\tau}-\Pi_{\mathcal{K}_{\tau}}\left(\xi_{\delta}\right)\right) \leq 0, \quad \xi_{\delta}^{\prime}\left(\xi_{\delta}-\Pi_{\mathcal{K}_{\delta}}\left(\xi_{\tau}\right)\right) \leq 0 \quad \text { a.e. in } Q
$$

where $\Pi_{\mathcal{K}_{\tau}}$ denotes the projection operator onto the convex $\mathcal{K}_{\tau}$. In particular, one obtains

$$
\left(\xi_{\tau}^{\prime}-\xi_{\delta}^{\prime}\right)\left(\xi_{\tau}-\xi_{\delta}\right) \leq-\xi_{\tau}^{\prime}\left(\xi_{\delta}-\Pi_{\mathcal{K}_{\tau}}\left(\xi_{\delta}\right)\right)-\xi_{\delta}^{\prime}\left(\xi_{\tau}-\Pi_{\mathcal{K}_{\delta}}\left(\xi_{\tau}\right)\right)
$$

and, integrating over $\Omega \times(0, t)$ for $t \in[0, T]$, we deduce that

$$
\frac{1}{2}\left\|\xi_{\tau}(t)-\xi_{\delta}(t)\right\|_{L^{2}(\Omega)}^{2} \leq \int_{Q}\left(\left|\xi_{\tau}^{\prime}\right|+\left|\xi_{\delta}^{\prime}\right|\right) d_{\mathbb{R}}\left(\mathcal{K}_{\tau}, \mathcal{K}_{\delta}\right) .
$$

By extracting some not relabeled pointwise converging subsequence from $\bar{w}_{\tau}$ we easily deduce

$$
\int_{\Omega} d_{\mathbb{R}}^{2}\left(\mathcal{K}_{\tau}, \mathcal{K}_{\delta}\right) \rightarrow 0 \quad \text { as } \tau, \delta \rightarrow 0 .
$$

In the latter we have also used the fact that we have

$$
\varepsilon\left(\bar{u}_{\tau}^{\text {Dir }}\right) \rightarrow \varepsilon\left(u^{\text {Dir }}\right) \quad \text { in } L^{\infty}\left(0, T ; H^{1}\left(\Omega ; \mathbb{R}_{\mathrm{sym}}^{3 \times 3}\right)\right) .
$$


We have hence proved that

$$
\left\|\xi_{\tau}-\xi_{\delta}\right\|_{C\left([0, T] ; L^{2}(\Omega)\right)} \rightarrow 0 \quad \text { as } \tau, \delta \rightarrow 0
$$

so that $\xi_{\tau}$ is a Cauchy sequence in $C\left([0, T] ; L^{2}(\Omega)\right)$. Moreover, we clearly have that

$$
\bar{\xi}_{\tau} \rightarrow \xi \quad \text { strongly in } L^{\infty}\left(0, T ; L^{2}(\Omega)\right),
$$

as well.

Finally, it remains to show that there exists $\sigma \in H^{1}\left(0, T ; L^{2}\left(\Omega ; \mathbb{R}_{\mathrm{sym}}^{3 \times 3}\right)\right)$ such that, up to a not relabeled subsequence, we have

$$
\sigma_{\tau}, \bar{\sigma}_{\tau} \rightarrow \sigma \quad \text { strongly in } L^{\infty}\left(0, T ; L^{2}\left(\Omega ; \mathbb{R}_{\mathrm{sym}}^{3 \times 3}\right)\right) .
$$

Indeed, our estimates yield

$$
\sigma_{\tau} \rightarrow \sigma \quad \text { weakly in } H^{1}\left(0, T ; L^{2}\left(\Omega ; \mathbb{R}_{\mathrm{sym}}^{3 \times 3}\right)\right),
$$

and we need to check that $\bar{\sigma}_{\tau}$ is a Cauchy sequence in $L^{\infty}\left(0, T ; L^{2}\left(\Omega ; \mathbb{R}_{\mathrm{sym}}^{3 \times 3}\right)\right)$. We can write

$$
\begin{aligned}
& \int_{\Omega}\left(\mu \mathrm{D} \varepsilon\left(\bar{w}_{\tau}\right) \because \mathrm{D} \varepsilon(v)+\bar{\sigma}_{\tau}: \varepsilon(v)\right)=\left\langle\widehat{f}_{\tau}, v\right\rangle \quad \forall v \in V, \text { a.e. in }(0, T), \\
& L \bar{\sigma}_{\tau}+\bar{\xi}_{\tau} \partial F\left(\bar{\sigma}_{\tau}\right) \ni \varepsilon\left(\bar{w}_{\tau}+\bar{u}^{\text {Dir }}\right) \quad \text { a.e. in } Q .
\end{aligned}
$$

By arguing similarly with respect to the proof of Theorem 4.1 (see estimate (4.13)), we easily deduce that

$$
\mu\left\|\mathrm{D} \varepsilon\left(\bar{w}_{\tau}-\bar{w}_{\delta}\right)\right\|_{L^{2}\left(\Omega ; \mathbb{R}^{3 \times 3 \times 3}\right)}+c\left\|\bar{\sigma}_{\tau}-\bar{\sigma}_{\delta}\right\|_{L^{2}\left(\Omega ; \mathbb{R}_{\mathrm{sym}}^{3 \times 3}\right)} \leq \frac{1}{c}\left\|\bar{\xi}_{\tau}-\bar{\xi}_{\delta}\right\|_{L^{2}(\Omega)} .
$$

5.3. Passage to the limit. We now prove that the triplet $(w, \sigma, \xi)$ solves the Quasistatic Evolution Problem. From the convergence (5.12) we deduce

$$
\begin{aligned}
& \mathrm{D} \varepsilon\left(w_{\tau}\right) \rightarrow \mathrm{D} \varepsilon(w) \quad \text { weakly in } H^{1}\left(0, T ; L^{2}\left(\Omega ; \mathbb{R}^{3 \times 3 \times 3}\right)\right), \\
& \mathrm{D} \varepsilon\left(\bar{w}_{\tau}\right) \rightarrow \mathrm{D} \varepsilon(w) \quad \text { weakly in } L^{2}\left(0, T ; L^{2}\left(\Omega ; \mathbb{R}^{3 \times 3 \times 3}\right)\right) .
\end{aligned}
$$

Hence, the equilibrium relation (3.7) follows. The constitutive relation (3.8) is obtained by simply passing to the limit in its discrete counterpart (5.19). For the proof of the dynamics (3.9), we first introduce the generalized play operator $\mathcal{P}$ such that $\xi(t)=\mathcal{P}\left[\varepsilon ; \xi_{0}\right](t)$, for every $t \in[0, T]$, where $\xi$ solves

$$
\dot{\xi}+\partial I_{\widehat{K}(\varepsilon)}(\xi) \ni 0, \quad \xi(0)=\xi_{0} .
$$

This mapping is Lipschitz continuous from $L^{2}\left(\Omega ; C\left([0, T] ; \mathbb{R}_{\mathrm{sym}}^{3 \times 3}\right)\right) \times[0,1]$ to $L^{2}(\Omega ; C([0, T]))$ with a Lipschitz constant given in terms of the Lipschitz constants of $\hat{\lambda}_{\ell}(\varepsilon), \hat{\lambda}_{u}(\varepsilon)$ (see [56]). Now, setting $\varepsilon_{\tau}:=\varepsilon\left(w_{\tau}\right)$, relation (5.15) yields

$$
\xi_{\tau}\left(t_{i}\right) \in \widehat{K}\left(\varepsilon_{\tau}\left(t_{i}\right)\right), \quad \dot{\xi}_{\tau}\left(t_{i}\right)\left(\xi_{\tau}\left(t_{i}\right)-v\right) \leq 0 \quad \forall v \in \widehat{K}\left(\varepsilon_{\tau}\left(t_{i}\right)\right)
$$

and therefore we have

$$
\xi_{i}=\xi_{\tau}\left(t_{i}\right)=\mathcal{P}\left[\varepsilon_{\tau} ; \xi_{0}\right]\left(t_{i}\right)=\bar{\xi}_{\tau}(t) \quad \forall t \in\left(t_{i-1}, t_{i}\right] .
$$

Using this, and also the fact that $\mathcal{P}$ is bounded also from $L^{2}\left(\Omega ; H^{1}\left(0, T ; \mathbb{R}_{\mathrm{sym}}^{3 \times 3}\right)\right) \times[0,1]$ to $L^{2}\left(\Omega ; H^{1}(0, T)\right)$, it is easy to prove that

$$
\left\|\bar{\xi}_{\tau}-\mathcal{P}\left[\varepsilon_{\tau} ; \xi_{0}\right]\right\|_{L^{2}\left(0, T ; L^{2}(\Omega)\right)} \leq C \tau
$$

Now observe that we have

$$
H^{1}\left(0, T ; H^{1}\left(\Omega ; \mathbb{R}_{\mathrm{sym}}^{3 \times 3}\right)\right)=H^{1}\left(\Omega ; H^{1}\left(0, T ; \mathbb{R}_{\mathrm{sym}}^{3 \times 3}\right)\right) \subset L^{2}\left(\Omega ; C\left([0, T] ; \mathbb{R}_{\mathrm{sym}}^{3 \times 3}\right)\right),
$$

with compact embedding, and therefore estimate (5.11) also implies that, up to a subsequence

$$
\varepsilon_{\tau} \rightarrow \varepsilon \quad \text { strongly in } L^{2}\left(\Omega ; C\left([0, T] ; \mathbb{R}_{\mathrm{sym}}^{3 \times 3}\right)\right),
$$


where $\varepsilon:=\varepsilon(w)$. Hence, by means of this last convergence, of the strong convergence (5.17) and of the above mentioned Lipschitz continuity property of $\mathcal{P}$, by passing to the limit as $\tau \rightarrow 0$ we conclude that

$$
\xi=\mathcal{P}\left[\varepsilon ; \xi_{0}\right]
$$

which means that $\xi$ and $\varepsilon$ solve (5.20) and the dynamics (3.9) of the single-variant volume fraction $\xi$ follows.

\section{ACKNOWLEDGMENTS}

US acknowledges partial support from FP7-IDEAS-ERC-StG Grant \#200497 (BioSMA) and the kind hospitality of the Weierstrass Institute for Applied Analysis and Stochastics in Berlin, where this project was initiated. The main part of this research has been performed during PK's visit in Pavia under the sponsorship of the Short-Term Mobility program of the CNR.

\section{REFERENCES}

[1] M. Arndt, M. Griebel and T. Roubícěk. Modelling and numerical simulation of martensitic transformation in shape memory alloys. Contin. Mech. Thermodyn., 15 (2003) 463-485.

[2] H. Attouch. Variational convergence for functions and operators, Pitman (Advanced Publishing Program), Boston, 1984.

[3] F. Auricchio. A robust integration-algorithm for a finite-strain shape-memory alloy superelastic model. Inter. J. Plast., 17 (2001) 971-990.

[4] F. Auricchio and J. Lubliner. A uniaxial model for shape-memory alloys. Internat. J. Solids Structures, 34 (1997) 3601-3618.

[5] F. Auricchio, A. Mielke, and U. Stefanelli. A rate-independent model for the isothermal quasi-static evolution of shape-memory materials, Math. Models Meth. Appl. Sci., 18 (2008) 1:125-164.

[6] F. Auricchio, A. Reali, and U. Stefanelli. A three-dimensional model describing stress-induces solid phase transformation with residual plasticity, Internat. J. Plast., 23 (2007) 2:207-226.

[7] F. Auricchio, A. Reali, and U. Stefanelli. A phenomenological 3D model describing stress-induced solid phase transformations with permanent inelasticity, in Topics on Mathematics for Smart Systems (Rome, 2006), 1-14, World Sci. Publishing, 2007.

[8] F. Auricchio, A. Reali, and U. Stefanelli. A three-dimensional model describing stress-induced solid phase transformation with permanent inelasticity, Internat. J. Plast., 23 (2007) 207-226.

[9] F. Auricchio, A. Reali, and U. Stefanelli. A macroscopic 1D model for shape memory alloys including asymmetric behaviors and transformation-dependent elastic properties, Comput. Methods Appl. Mech. Engrg., 198 (2009) 1631-1637.

[10] F. Auricchio and U. Stefanelli, Numerical analysis of a 3D super-elastic constitutive model. Internat. J. Numer. Meth. Engrg., 61 (2004) 142-155.

[11] F. Auricchio and U. Stefanelli. Well-posedness and approximation for a one-dimensional model for shape memory alloys. Math. Models Meth. Appl. Sci., 15 (2005) 9:1301-1327.

[12] F. Auricchio, R.L. Taylor and J. Lubliner. Shape-memory alloys: macromodelling and numerical simulations of the superelastic behavior. Comput. Methods Appl. Mech. Engrg., 146 (1997) 281-312.

[13] C. Baiocchi and A. Capelo. Variational and quasivariational inequalities, applications to free boundary problems. Wiley, Chichester, 1983.

[14] V. Barbu. Nonlinear semigroups and differential equations in Banach spaces. Noordhoff, Leyden, 1976.

[15] M. Brokate and J. Sprekels. Hysteresis and phase transitions, vol. 121 of Applied Mathematical Sciences, Springer-Verlag, New York, 1996.

[16] P. Colli, M. Frémond and A. Visintin. Thermo-mechanical evolution of shape memory alloys. Quart. Appl. Math., 48 (1990) 31-47.

[17] P. Colli and J. Sprekels. Global existence for a three-dimensional model for the thermodinamical evolution of shape memory alloys. Nonlinear Anal., 18 (1992) 873-888.

[18] G. Dal Maso, A. DeSimone, and M. G. Mora. Quasistatic evolution problems for linearly elastic-perfectly plastic materials. Arch. Rational Mech. Anal., 180 (2006) 2:237-291.

[19] T. W. Duerig, K. N. Melton, D. Stökel, C. M. Wayman editors. Engineering aspects of shape memory alloys, Butterworth-Heinemann, 1990.

[20] T. W. Duerig, A. R. Pelton editors. SMST-2003 Proceedings of the International Conference on Shape Memory and Superelastic Technology Conference, ASM International, 2003. 
[21] G. Duvaut and J.-L. Lions. Inequalities in Mechanics and Physics. Springer, Berlin, 1976.

[22] F. Falk, Martensitic domain boundaries in shape-memory alloys as solitary waves, J. Phys. C4 Suppl., 12 (1982) 3-15.

[23] F. Falk and P. Konopka. Three-dimensional Landau theory describing the martensitic phase transformation of shape-memory alloys, J. Phys. Condens. Matter, 2 (1990) 61-77.

[24] G. Francfort and A. Mielke. Existence results for a class of rate-independent material models with nonconvex elastic energies. J. Reine Angew. Math., 595 (2006) 55-91.

[25] M. Frémond. Matériaux à mémoire de forme, C. R. Acad. Sci. Paris Sér. II Méc. Phys. Chim. Sci. Univers Sci. Terre, 304 (1987) 239-244.

[26] M. Frémond. Non-smooth Thermomechanics. Springer-Verlag, Berlin, 2002.

[27] S. Govindjee, C. Miehe. A multi-variant martensitic phase transformation model: formulation and numerical implementation, Comput. Methods Appl. Mech. Engrg., 191 (2001) 215-238.

[28] W. Han, B.D. Reddy. Plasticity, Mathematical theory and numerical analysis. Springer-Verlag, New York, 1999.

[29] D. Helm and P. Haupt. Shape memory behaviour: modelling within continuum thermomechanics, Internat. J. Solids Structures, 40 (2003) 827-849.

[30] M. A. Krasnosel'skii and A. V. Pokrovskii, Systems with Hysteresis., Springer, Berlin (1989).

[31] P. Krejčí and U. Stefanelli. Existence and nonexistence for the full thermomechanical Souza-Auricchio model of shape memory wires. Submitted, 2009.

[32] P. Krejčí and U. Stefanelli. Well-posedness of a thermo-mechanical model for shape memory alloys under tension. Submitted, 2009.

[33] M. Kružík, A. Mielke, and T. Roubíček. Modelling of microstructure and its evolution in shape-memory-alloy single cristals, in particular in CuAlNi. Meccanica, 40 (2005) 4-6:389-418.

[34] D. C. Lagoudas, P. B. Entchev, P. Popov, E. Patoor, L. C. Brinson, X. Gao. Shape memory alloys, Part II: Modeling of polycrystals, Mech. Materials, 38 (2006) 391-429.

[35] V.I . Levitas. Thermomechanical theory of martensitic phase transformations in inelastic materials, Internat. J. Solids Structures, 35 (1998) 889-940.

[36] J. Lubliner and F. Auricchio. Generalized plasticity and shape-memory alloys. Internat. J. Solids Structures, 33 (1996) 991-1003.

[37] A. Mielke. Evolution of rate-independent systems. In C. Dafermos and E. Feireisl, editors, Handbook of Differential Equations, Evolutionary Equations, Vol 2, Elsevier B.V. 2005, 461-559.

[38] A. Mielke and T. Roubíček. A rate-independent model for inelastic behavior of shape-memory alloys. Multiscale Model. Simul., 1 (2003) 4:571-597.

[39] A. Mielke, T. Roubíček, and U. Stefanelli. Г-convergence and relaxations for rate-independent evolution equations. Calc. Var. Partial Differential Equations, 31 (2008) 3:387-416.

[40] A. Mielke and F. Theil. On rate-independent hysteresis models. NoDEA, Nonlinear Diff. Equations Applications, 11 (2004) 151-189.

[41] A. Mielke, F. Theil and V.I. Levitas. A variational formulation of rate-independent phase transformations using an extremum principle. Arch. Ration. Mech. Anal., 162 (2002) 137-177.

[42] M.D.P. Monteiro Marques, Differential inclusions in nonsmooth mechanical problems- shocks and dry friction. Birkhaüser, Basel Boston Berlin 1993.

[43] J.-J. Moreau, Problème d'évolution associé à un convexe mobile d'un espace hilbertien. C. R. Acad. Sci. Paris Sér. A-B, 276, A791-A794, 1973.

[44] J.-J. Moreau, Evolution problem associated with a moving convex set in a Hilbert space. J. Differential Equations, 26, 3:347-374, 1977.

[45] U. Mosco. Convergence of convex sets and of solutions of variational inequalities, Advances in Math., 3 (1969) $510-585$.

[46] B. Peultier, T. Ben Zineb, and E. Patoor. Macroscopic constitutive law for SMA: Application to structure analysis by FEM, Materials Sci. Engrg. A, 438-440 (2006) 454-458.

[47] R. Peyroux, A. Chrysochoos, Ch. Licht, and M. Löbel. Phenomenological constitutive equations for numerical simulations of SMA's structures. Effect of thermomechanical couplings, J. Phys. C4 Suppl., 6 (1996) 347-356.

[48] P. Popov, D. C. Lagoudas. A 3-D constitutive model for shape memory alloys incorporating pseudoelasticity and detwinning of self-accommodated martensite, Internat. J. Plast., 23 (2007) 1679-1720.

[49] B. Raniecki and Ch. Lexcellent. $R_{L}$ models of pseudoelasticity and their specification for some shape-memory solids, European J. Mech. A Solids, 13 (1994) 21-50.

[50] S. Reese and D. Christ. Finite deformation pseudo-elasticity of shape memory alloys - Constitutive modelling and finite element implementation, Internat. J. Plast., 28 (2008) 455-482.

[51] R. Rossi and U. Stefanelli An order approach to a class of quasivariational sweeping processes. Adv. Differential Equations, 10 (2005), 5:527-552. 
[52] T. Roubíček. Evolution model for martensitic phase transformation in shape-memory alloys. Interfaces Free Bound. 4 (2002) 2:111-136.

[53] T. Roubíček, Models of microstructure evolution in shape memory alloys, in Nonlinear Homogenization and its Appl.to Composites, Polycrystals and Smart Materials, P. Ponte Castaneda, J. J. Telega, B. Gambin eds., NATO Sci. Series II/170, Kluwer, Dordrecht, 2004, pp.269-304.

[54] P. Thamburaja and L. Anand. Polycrystalline shape-memory materials: effect of crystallographic texture, $J$. Mech. Phys. Solids, 49 (2001) 709-737.

[55] F. Thiebaud, Ch. Lexcellent, M. Collet, and E. Foltete. Implementation of a model taking into account the asymmetry between tension and compression, the temperature effects in a finite element code for shape memory alloys structures calculations, Comput. Materials Sci., 41 (2007) 2: 208-221.

[56] A. Visintin. Differential Models of Hysteresis, vol. 111 of Applied Mathematical Sciences, Springer, Berlin, 1994.

IMATI - CNR, Via FERrata 1, I-27100 PAVia, Italy

E-mail address: sergio.frigeri@imati.cnr.it

$U R L:$ http://www.imati.cnr.it/frigeri/

MatematickÝ ústav AV ČR, Žitná 25, CZ-11567 Praha 1, Czech Republic

E-mail address: krejci@math.cas.cz

$U R L:$ http://www.math.cas.cz/ ${ }^{\mathrm{krej}} \mathrm{ci} /$

IMATI - CNR, VIA Ferrata 1, I-27100 PAVia, ItAly

E-mail address: ulisse.stefanelli@imati.cnr.it

URL: http://www.imati.cnr.it/ulisse 